\title{
Exploration of surfaces by atomic scattering in the almost classical regime
}

\author{
K. Burke ${ }^{1}$, J.H. Jensen ${ }^{2}$ and W. Kohn \\ Department of Physics, University of California, Santa Barbara, CA 93106, USA
}

Received 2 February 1990; accepted for publication 14 August 1990

\begin{abstract}
Inelastic atom-surface scattering at hyperthermal $(-1 \mathrm{eV})$ energies is discussed. In this regime, the scattering is almost classical. However, for a surface at low temperature, peaks in the final energy distribution are broadened by quantum effects. The widths of these peaks provide a direct measure of the zero point motion of the surface atoms. For impulsive collisions, we derive a simple expression for these widths. As an example, we present a semi-quantitative analysis for He striking a LiF surface, using Born-Mayer forces between the atoms to estimate the amplitude of the zero point fluctuations. We discuss, in detail, how single peaks may be isolated in an experiment. Measurements of this effect could provide an important probe of surface dynamics.
\end{abstract}

\section{Introduction}

The present state of the art of atom surface experiments allows the production of highly monoenergetic incident beams $(\Delta E / E=1 \%)[1]$, and the resolution of both the angular and the energy distributions of scattered beams [2]. At thermal energies in particular, theory has followed experiment closely, and there are now many calculations which yield good agreement with experiment for both elastic [3] and inelastic [4] scattering. For example, surface phonon dispersion curves have been derived by using energy and momentum conservation alone, from studies of one-phonon events which are prominent at these energies [2]. From an analysis of experiments in this energy regime, dominated by quantum effects, a variety of parameters describing the surface structural properties [5], surface lattice dynamics [2] and the atom-surface potential [6] have been deduced.

'Present address: Department of Physics and Astronomy, Rugters University, P.O. Box 849, Piscataway, NJ 088550849, USA.

2 Present address: Department of Physics and Astronomy, University of Maryland, College Park, MD 20742, USA.
There are no major experimental difficulties [7] in working with high quality beams at energies up to a few $\mathrm{eV}$, where quantum effects are rather small. Experiments at these energies in principle contain information about the motion of individual atoms, as opposed to the global information contained in experiments with low energy atoms of long Debye wavelength. However, theoretical analysis of such experiments has been limited to either purely classical calculations [8], or partially quantum mechanical calculations [9]. In general, the relation between surface features and the final energy and angular distributions of the scattered atoms is quite complex, and most such calculations require considerable numerical work to make a realistic comparison with experiment [10].

In this paper, we show that inelastic scattering at hyperthermal energies can yield a direct measure of the quantum zero point motions of atoms at the surface of a crystal. To illustrate this effect we consider first classical elastic scattering at normal incidence from a rigid one-dimensional surface at zero temperature (see fig. 1). The beam particles are reflected at the classical turning points of the atom-surface potential for the given incident energy. For simplicity, we have chosen a curve with 


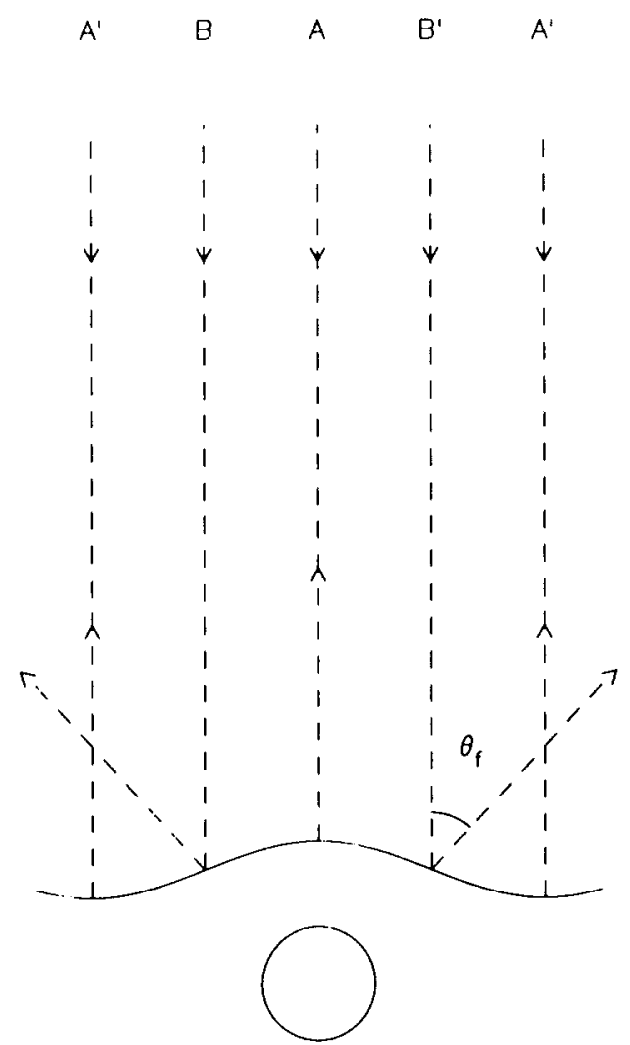

Fig. 1. Classical elastic scattering from a one-dimensional monatomic surface at zero temperature. The solid line is an equipotential curve along which the potential energy equals the initial energy of the incident particle.

a corrugation small enough to avoid multiple scattering events. For this case, a detector in the scattering direction $\theta_{\mathrm{f}}=0$ (beams $A$ and $A^{\prime}$ ) sees only particles impacting directly on a local maximum or minimum of the turning point curve. Under inelastic scattering conditions with target temperature $T=0$, each impact parameter (distance from beam $A$ ) will still give rise to a unique scattering angle, $\theta_{\mathrm{f}}$, and final energy $E_{\mathrm{f}}$. In particular, normal scattering $\left(\theta_{\mathrm{f}}=0\right)$ will still come only from beams $A$ and $A^{\prime}$ but, because of the physical difference between the two impact points, there will be two different final energies, $E_{\mathrm{f}}$ and $E_{\mathrm{f}}^{\prime}$, associated with $\theta_{\mathrm{f}}=0$. Thus the final energy spectrum consists of two delta function peaks at energies less than the initial energy. Lastly, we consider the corrections to this picture due to quantum mechanics. Fig. 2 is a schematic of how the final energy distribution appears. Atoms scattered in the direction $\theta_{f}=0$ will have an energy distribution consisting of two peaks whose finite widths reflect the zero point motion of the atoms.

Broadening of loss spectrum peaks is a wellknown phenomenon in ion-scattering [11]. Unfortunately, the ability to detect and measure zero point motion is limited by the minimum energies achievable with ion beams [12]. Böheim and Brenig [13] have discussed this "quantum broadening" for atom-scattering and noted its origin in zero point vibrations. However, they consider only flat surfaces, which yield a single peak in the final encrgy spectrum. Furthcrmore, in thcir calculation of the magnitude of this effect, they use the trajectory approximation [9], which has been shown to be inaccurate under many conditions [14]. We employ a more recent theory whose range of validity is much broader and is better understood [14]. In this paper we show how these "quantum" widths may be calculated for any surface whose vibrational properties and interaction with an inci-

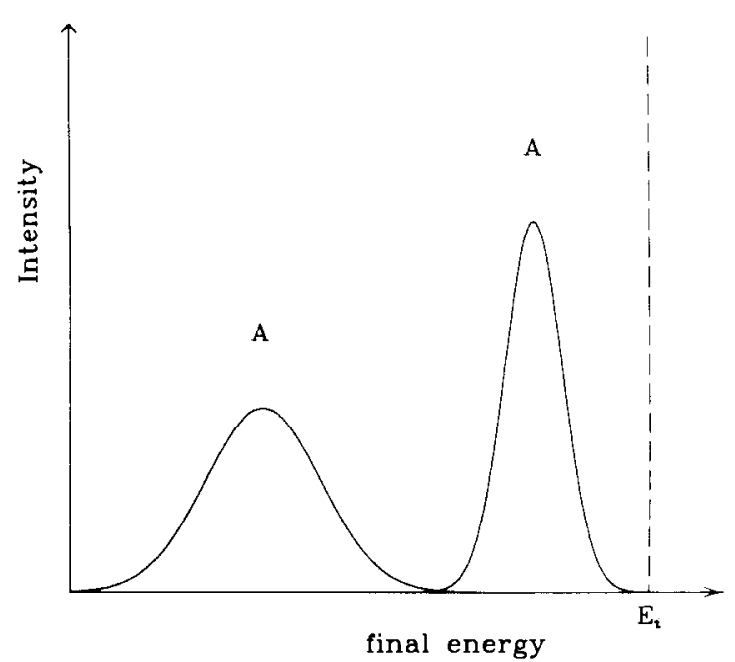

Fig. 2. Schematic final energy spectrum for quantum mechanical inelastic scattering at normal incidence and reflection from the surface shown in fig. 1. 
dent atom are known. Thus, the widths of these peaks give detailed information about the dynamics of the individual surface atoms.

For impulsive scattering from a single surface atom, the calculation becomes extremely simple, and an analytic result is given. For a real corrugated surface there will generally be several peaks in the final energy spectrum, but by careful consideration of the mapping of impact parameters to final momenta, we show how the final energy distribution reduces to a single peak for some scattering conditions. For a prototype experiment, we consider noble gas scattering from alkali halide surfaces, as the atom-surface potential and zero point fluctuations of the surface atoms may be theoretically estimated for these systems. As an illustration, we study the case of He striking a $\mathrm{LiF}$ surface in detail; we choose this system both to minimize errors made in our approximations and because it offers a simple final energy spectrum. However, we provide sufficient information to repeat the calculation for other noble gas-alkali halide systems, and our analytic expression may also be useful in the study of more complicated surfaces. We use our model to show how the widths depend on the incident and final directions, the incident energy and the ratio of the masses of the incident and surface atoms. We also discuss how these widths are increased at finite surface temperatures.

In section 2 we present the semiclassical result which is the basis of our approach. We also derive a simple expression for the width when the incident particle is scattered impulsively from a single surface atom. Section 3 describes the calculation of surface properties needed to make predictions for the widths, viz. the atom-surface potential and the zero-point fluctuations of surface atoms. In section 4 we present results for a prototype system: He in-plane scattering from the $\mathrm{LiF}(001)$ surface at $0.5 \mathrm{eV}$ energy and indicate how a simple final energy spectrum may be obtained. In section 5 , we consider the temperature dependence of the peak widths and suggest an experiment to test our predictions. The last section gives our conclusions. The appendix consists of a description of how the surface vibrational amplitudes were estimated using Born-Mayer [15] potentials.

\section{Calculation of widths}

This section is devoted to calculating the widths of energy peaks when the collision is impulsive, and the incident particle interacts with only onc surface atom. The starting point of our calculation is a result derived in recent work of Jensen et al. [14]. In this work, a semiclassical theory of inelastic scattering for low surface temperatures is derived by expanding quantum mechanical expectation values in powers of $\hbar$. The lowest order contribution to the square uncertainty of an energy peak distribution for a particular final scattered direction is:

$$
\begin{aligned}
(\Delta E)^{2}- & \sum_{i, j}\left(\frac{\partial E_{\mathrm{f}}}{\partial X_{i}}\right)\left(\frac{\partial E_{\mathrm{f}}}{\partial X_{j}}\right)\left\langle\hat{X}_{i} \hat{X}_{j}\right\rangle \\
& +\sum_{i, j}\left(\frac{\partial E_{\mathrm{f}}}{\partial P_{i}}\right)\left(\frac{\partial E_{\mathrm{f}}}{\partial P_{j}}\right)\left\langle\hat{P}_{i} \hat{P}_{j}\right\rangle
\end{aligned}
$$

Here $E_{\mathrm{f}}$ is the classical final energy; $X_{i}$ and $P_{i}$ are the position (measured from equilibrium) and momentum coordinates of an atom in the crystal, while $i$ and $j$ label all spatial atomic coordinates ( $3 N$ for a crystal of $N$ atoms). The expectation values are to be evaluated quantum mechanically for the undisturbed crystal. The derivatives are of the classical final energy with respect to initial values for the surface position and momentum coordinates, and are to be evaluated at the classical equilibrium values of the coordinates. The incident and final directions and the initial energy of the scattered particle are held fixed in these derivatives. $\Delta E$ is the width of the peak, defined by the relation

$$
(\Delta E)^{2}=\frac{\int I(E)(E-\bar{E})^{2} \mathrm{~d} E}{\int I(E) \mathrm{d} E},
$$

where $I(E)$ is the intensity and $\bar{E}$ is the mean energy of the peak. For a harmonic crystal, the quantum expectation values appearing in eq. (1) are proportional to $\hbar$, so that $\Delta E \sim \hbar^{1 / 2}$, and eq. (1) is exact to this order [16]. 
Eq. (1) is a prescription for calculating the width of an energy peak in the final energy spectrum. It is useful because it requires knowledge of only classical solutions of scattering problems and of the quantum mechanical surface dynamics in the absence of the scattering atom, both of which have been studied in detail for many different systems [17]. Higher order corrections are negligible if $\hbar \omega_{\mathrm{D}}$ is much smaller than $E_{\text {loss }}$, where $\omega_{\mathrm{D}}$ is the Debye frequency of the crystal and $E_{\text {loss }}$ is the classical energy loss of the incident particle due to the collision. For typical solids [18] $\hbar \omega_{\mathrm{D}}$ is of order $50 \mathrm{meV}$ so that a $0.5 \mathrm{eV}$ beam which loses a substantial fraction of its energy on striking a surface, easily satisfies this condition. At these energies, the incident particle interacts strongly with only a small number of surface atoms ncar the impact point, so that in practice relatively few derivatives need to be summed over.

We now apply this formula under very specific assumptions which allow a simple analytic expression for the width of a peak. To begin with, we assume that the incident atom interacts with only one surface atom, which we call the target atom; secondly, that the collision is so abrupt that the restoring forces on the surface atom have negligible effect on the collision; and finally, we consider only those collisions in which the incident atom does not strike the target atom more than once in the course of the scattering process. The first of these conditions precludes scattering from any point on the surface where more than one atom makes a major contribution to the potential. Thus we would not be able to find the widths of the peaks due to scattering from the valleys between atoms in fig. 1. The second condition is called the impulse approximation, as the energy exchange during the collision occurs too quickly for the lattice forces to have appreciable effects. The last condition, combined with the first two, restricts us to cases where the incident mass is less than the mass of the target atom, the ensure that the incident atom is moving away from the surface after the collision.

For the above conditions, the calculation itself is simple. The incident atom strikes a single surface atom which acts like a free particle. For a two-particle free collision, energy and momentum con- servation yicld, for the magnitude of the final momentum of the incident particle, in a given final direction

$$
\begin{aligned}
p_{\mathrm{f}}= & \left\{\mu\left[\boldsymbol{p}_{\mathrm{i}}+\boldsymbol{P}\right] \cdot \boldsymbol{n}_{\mathrm{f}}+\left(\left[\left(\boldsymbol{p}_{\mathrm{i}}+\boldsymbol{P}\right) \cdot \boldsymbol{n}_{\mathrm{f}}\right]^{2} \mu^{2}\right.\right. \\
& \left.\left.+[1+\mu]\left[(1-\mu) p_{\mathrm{i}}^{2}-2 \mu \boldsymbol{p}_{\mathrm{i}} \cdot \boldsymbol{P}\right]\right)^{1 / 2}\right\} \\
& \times(1+\mu)^{-1}
\end{aligned}
$$

In this expression $\mu=m_{\mathrm{i}} / m_{\mathrm{s}}$, the ratio of the incident atom's mass to that of the surface atom: $\boldsymbol{P}$ is the initial momentum of the surface atom and $p_{i}$ and $p_{\mathrm{f}}$ are the initial and final momenta of the incident particle; and, for any vector $\boldsymbol{p}, p=|\boldsymbol{p}|$ and $n=p / p$. As $E_{\mathrm{f}}=p_{\mathrm{f}}^{2} / 2 m_{\mathrm{i}}$, we see that $E_{\mathrm{f}}$ is independent of the initial position of the target atom (for a fixed final direction), so that, from eq. (1), only momentum fluctuations contribute. Substituting both eq. (3) and its first derivative with respect to $\boldsymbol{P}$ at $\boldsymbol{P}=\mathbf{0}$ into eq. (1), we find, for the conditions stated above,

$$
\begin{aligned}
& E_{\mathrm{f}}=f\left(\boldsymbol{n}_{\mathrm{i}} \cdot \boldsymbol{n}_{\mathrm{f}}\right) E_{\mathrm{i}}, \\
& (\Delta E)^{2}=E_{\mathrm{i}} \sum_{\alpha, \beta} g_{\alpha \beta}\left(\boldsymbol{n}_{\mathrm{i}}, \boldsymbol{n}_{\mathrm{f}}\right) \frac{2\left\langle\hat{P}_{\alpha} \hat{P}_{\beta}\right\rangle}{m_{\mathrm{s}}},
\end{aligned}
$$

where

$$
\begin{aligned}
& f\left(\boldsymbol{n}_{\mathrm{i}} \cdot \boldsymbol{n}_{\mathrm{f}}\right) \\
& =\left[\frac{\mu\left(\boldsymbol{n}_{\mathrm{i}} \cdot \boldsymbol{n}_{\mathrm{f}}\right)+\sqrt{\left(\boldsymbol{n}_{\mathrm{i}} \cdot \boldsymbol{n}_{\mathrm{f}}\right)^{2} \mu^{2}+1-\mu^{2}}}{1+\mu}\right]^{2}, \\
& g_{\alpha \beta}\left(\boldsymbol{n}_{\mathrm{i}}, \boldsymbol{n}_{\mathrm{f}}\right)=\left[\frac{f\left(\boldsymbol{n}_{\mathrm{i}} \cdot \boldsymbol{n}_{\mathrm{f}}\right)}{\mu}\right]\left(\frac{\partial p_{\mathrm{f}}}{\partial P_{\alpha}}\right)_{\boldsymbol{P}=\mathbf{0}}\left(\frac{\partial p_{\mathrm{f}}}{\partial P_{\beta}}\right)_{\boldsymbol{P}=\mathbf{0}},
\end{aligned}
$$

and

$$
\begin{aligned}
\left(\frac{\partial p_{\mathrm{f}}}{\partial \boldsymbol{P}}\right)_{\boldsymbol{P}=\mathbf{0}}= & \left(\frac{\mu}{1+\mu}\right) \\
& \times\left[\boldsymbol{n}_{\mathrm{f}}+\frac{\mu\left(\boldsymbol{n}_{\mathrm{i}} \cdot \boldsymbol{n}_{\mathrm{f}}\right) \boldsymbol{n}_{\mathrm{f}}-(1+\mu) \boldsymbol{n}_{\mathrm{i}}}{\sqrt{\left(\boldsymbol{n}_{\mathrm{i}} \cdot \boldsymbol{n}_{\mathrm{f}}\right)^{2} \mu^{2}+1-\mu^{2}}}\right] .
\end{aligned}
$$

Here $\alpha$ and $\beta$ denote the Cartesian components of the surface atom. The function $f$ and $g_{\alpha \beta}$ are purely kinematic factors depending only on $\boldsymbol{n}_{\mathrm{i}}$ 
and $\boldsymbol{n}_{\mathrm{f}}$. These equations are universal, as they apply to the scattering of any atom from any crystal surface, once the above conditions are met. We refer to such single impulsive two-body collisions as simple collisions. We note also that the atom-surface interaction does not enter these equations, and that its sole relevance is in determining when a collision is simple.

\section{Simple collisions}

\subsection{The atom-surface interaction}

In this section, we give a simple prescription for estimating the atom-surface potential at hyperthermal energies for noble gases interacting with alkali halide surfaces. The fundamental assumption is that the potential may be written as the sum of pair potentials between the incident particle and each surface atom [19]. This assumption works well for species with closed electronic shells, as is the case here [20]. We further assume that these pair potentials are the same as those found in ion beam experiments, where a beam of either alkali or halide ions collides with a neutral noble gas. Such potentials can be very well approximated (in this regime) by a simple exponential repulsion [21]:

$V(r)=V_{0} \exp (-r / d)$,

where $r$ is the separation distance and $V_{0}$ and $d$ are two empirical parameters which depend on the species involved. We emphasize that eq. (9) is an empirical approximation to the total potential, rather than just the repulsion due to electronic wavefunction overlap. However the effect of the (relatively) small attractive well is negligible at these large incident and outgoing energies. We call $V_{0}$ the strength of the repulsion and $d$ its range. Values for the strengths and ranges from many closed-shell species interactions are available from both ab initio calculations (e.g., the work of Gordon and Kim [22]) and from beam experiments (e.g., the work of Kita, Noda and Inouye [21]). Table 1 lists some of these values, and they are used to plot the equipotential surfaces shown in fig. 3. These are defined by

$V(\boldsymbol{r})=E_{\mathrm{i}}$,

where $V(r)$ is the total potential felt by the incident atom at a position $r$, when the surface atoms are in their equilibrium positions. The first surface is for He interacting with $\mathrm{LiF}$ with $E_{\mathrm{i}}=0.5 \mathrm{eV}$, the second being the $\mathrm{He}-\mathrm{CsF}$ potential at the same energy. In fig. $3 a$, we see that the potential surface is only slightly convex over a $\mathrm{Li}$ atom. This shows that the $\mathrm{F}$ atoms nearest to a $\mathrm{Li}$ atom make a substantial contribution to the potential at this point. Thus the width of the peak due to a collision with the $\mathrm{Li}$ atom cannot be estimated using our simple expression.

\subsection{Surface vibrational properties}

Many calculations of surface phonon spectra and polarizations show excellent agreement with the results of low energy experiments [4]. Such calculations could be used to calculate the expressions $\left\langle\hat{X}_{i} \hat{X}_{j}\right\rangle$ and $\left\langle\hat{P}_{i} \hat{P}_{j}\right\rangle$ in eq. (1). However, crude estimates for these quantities are more easily obtained by assuming that an atom in an alkali halide crystal may be treated as an anisotropic Einstein oscillator. For collisions with such an
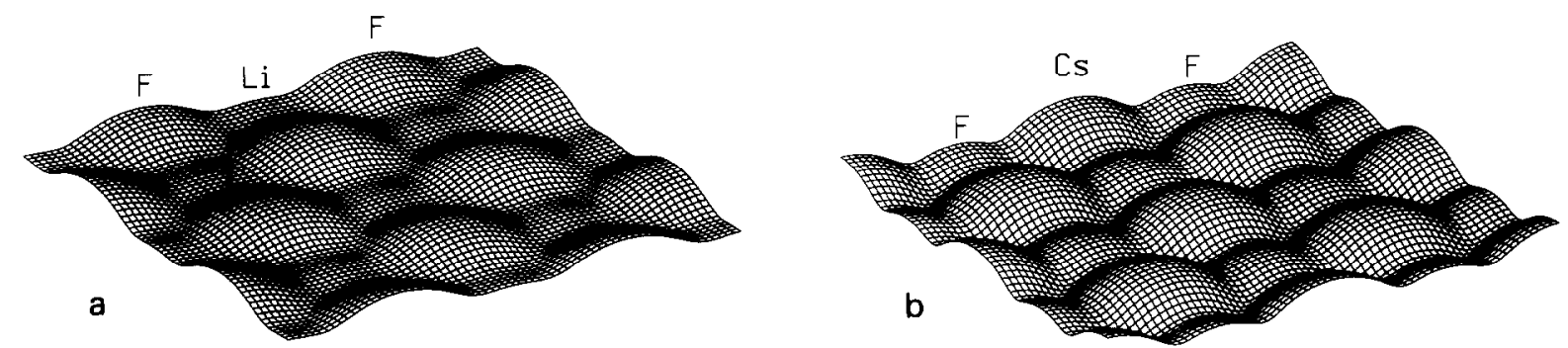

Fig. 3. Equipotential surfaces for two different systems. The first is $\mathrm{He}-\mathrm{LiF}$ at $0.5 \mathrm{eV}$, the second is $\mathrm{He}-\mathrm{CsF}$ at $0.5 \mathrm{eV}$. The interatomic spacing in (a) is $2.01 \AA$ while that of (b) is $3.00 \AA$. 
Table 1

Pair potential parameters used to construct fig. 3

\begin{tabular}{llll}
\hline Atom & Ion & \multicolumn{1}{l}{$\begin{array}{l}V_{0} \\
(\mathrm{eV})\end{array}$} & $\begin{array}{l}d \\
(\AA)\end{array}$ \\
\hline $\mathrm{He}$ & $\mathrm{Li}^{+}$ & 428 & $0.20^{\mathrm{a})}$ \\
$\mathrm{He}$ & $\mathrm{F}^{-}$ & 560 & $0.25^{\mathrm{b})}$ \\
$\mathrm{He}$ & $\mathrm{Cs}^{+}$ & 1380 & $0.28^{\mathrm{c})}$ \\
$\mathrm{Ne}$ & $\mathrm{Cs}^{+}$ & 5580 & $0.26^{\mathrm{d})}$ \\
\hline
\end{tabular}

a) Ref. [30].

b) Ref. [31].

c) Ref. [32].

d) Ref. [33].

atom at zero temperature, the expectation value appearing in eq. (5) becomes:

$$
\left\langle\hat{P}_{\alpha} \hat{P}_{\beta}\right\rangle=\delta_{\alpha \beta}\left(\frac{1}{2} m_{\mathrm{s}} \hbar \omega_{\alpha}\right) \text {, }
$$

where $\omega_{\alpha}$ is the frequency in the $\alpha$-direction. The frequencies are determined by the harmonic restoring forces felt by the atom when it is displaced slightly from equilibrium. We use the Born-Mayer scheme for estimating these forces. This calculation is straightforward and is described in the appendix; here we simply give expression for the frequencies of oscillations perpendicular and parallel to the surface of a crystal with the $\mathrm{NaCl}$ structure

$\omega_{\perp}=\left(\frac{\omega_{\mathrm{B}}}{\sqrt{2}}\right) \sqrt{\left(a / \rho+c_{\perp}\right) /(a / \rho-2)}$

$\omega_{\|}=\omega_{\mathrm{B}} \sqrt{\left(a / \rho-c_{\|}\right) /(a / \rho-2)}$.

Here $\omega_{B}$ is the effective Einstein frequency for a bulk atom, $\rho$ is the decay length of the BornMayer repulsion and $a$ is the interatomic spacing. The constants $c_{\perp}$ and $c_{\|}$are aproximately 0.542 and 2.636, respectively. Both $\rho$ and $\omega_{\mathrm{B}}$ are expressed in terms of the bulk modulus of the crystal and the interatomic spacing in the appendix. We may also calculate the corresponding mean squarc velocities of the zero point motion. These agree to within $20 \%$ with values reported for six alkali halide crystals, obtained from more sophisticated calculations [23]. The values for the frequencies are listed in table 2. Where available, we use the results of the more sophisticated calculations for estimating peak widths in the remainder of this work.

\subsection{Simple collisions}

With our estimates for the interaction and vibrational frequencies, we can now determine if a collision is simple. We demonstrate this for our prototype, $\mathrm{He}$ on $\mathrm{LiF}(001)$ at $0.5 \mathrm{eV}$. For the experimental repulsion given by eq. (9), a measure of the time spent in the vicinity of the surface atom, $\tau$, is of the order $d / v_{\mathrm{i}}+d / v_{\mathrm{f}}$, where $v_{\mathrm{i}}$ and $v_{\mathrm{f}}$ are the initial and final velocities of the incident particle, respectively. If $\omega$ is a frequency characteristic of the surface atom's motion, then a collision is impulsive if $\omega \tau \ll 1$. For normal incidence and detection, we find $\omega \tau=0.4$ for the collision with the $\mathrm{F}$ atom. We performed numerical calculations to check that the value was sufficiently small. We assumed that the incident particle interacts with only one atom (the $F$ atom) and that the target atom is an Einstein oscillator. For this scattering geometry, the problem becomes one-dimensional, and only the perpendicular frequency is relevant. Both the width and the classical final energy were found to agree with the impulsive results to within $15 \%$.

For He striking $F$, the mass ratio $\mu$ is small $(\approx 0.2)$ and one finds that only a single collision takes place during the scattering process. Note that for many other systems (e.g., with larger mass ratios or with less impulsive collisions) multiple collisions must be accounted for [24]. Finally, we must check that the effect of neighboring atoms on the final energy is negligible. This will depend on the particular trajectory considered. For example, for normal incidence and detection after

Table 2

Frequencies associated with various alkali halide crystals (all in units of $10^{13} \mathrm{rad} / \mathrm{s}$ )

\begin{tabular}{llccc}
\hline Atom & Crystal & $\omega_{\perp}$ & $\omega_{\|}$ & $\omega_{\mathrm{B}}$ \\
\hline $\mathrm{Li}$ & $\mathrm{LiF}$ & 7.28 & 7.79 & 8.35 \\
& & $(6.42)$ & $(6.64)$ & $(7.16)$ \\
$\mathrm{F}$ & $\mathrm{LiF}$ & 4.42 & 4.73 & 5.07 \\
& & $(4.13)$ & $(4.80)$ & $(5.16)$ \\
$\mathrm{Cs}$ & $\mathrm{CsF}$ & 1.11 & 1.33 & 1.38 \\
$\mathrm{~F}$ & $\mathrm{CsF}$ & 2.94 & 3.51 & 3.65 \\
\hline
\end{tabular}

The three columns of figures give the surface and bulk frequencies. The figures in brackets are calculated from values given by Chen et al. [23]. 
scattering from a $F$ atom, the turning point of the incident $\mathrm{He}$ atom will be about $1.7 \AA$ above the equilibrium position of the $F$ atom. At this point the force on the $\mathrm{He}$ atom due to neighboring atoms is only $1 \%$ of the force exerted by the target atom. Thus, such a collision is "simple".

\section{Results}

Before considering a realistic two-dimensional surface in detail, we return briefly to the schematic one-dimensional surface in fig. 1 to illustrate a few key features. For general inelastic scattering from such a surface, the final energy spectrum may be quite complicated. As a guide to the number and position of final energy peaks, we consider classical scattering from a rigid hard surface positioned at the equipotential curve [eq. 101. To begin with, we note that for a sufficiently smooth surface and small angle of incidence, neither shadowing nor multiple collisions will occur, i.e., there will be a one-to-one correspondence between impact parameters and points on the surface. Under these circumstances, the total scattering angle will be simply twice the angle between the incident beam and the surface normal at the point of impact. Thus, for any given incident angle, the direction of the surface normal uniquely determines the final scattered direction. If, for example, we fix the total scattering angle to be zero, so that we detect only particles directly backscattered from the surface, then the beam points along the normal at the impact point and, by rotating the direction of incidence, we can usually detect scattering from exactly two distinct points on the surface. However, as we reach the angle corresponding to the point of inflection of the surface, these two impact points coincide (see fig. 4). The intensity becomes singular at this angle, and, for larger incident angles, it vanishes entirely. This is known as a classical rainbow [10] and an angle at which the intensity becomes singular is called a rainbow angle. For classical inelastic scattering from a realistic potential, there will also be a maximum angle beyond which no scattering can occur, and at this angle, only one peak will

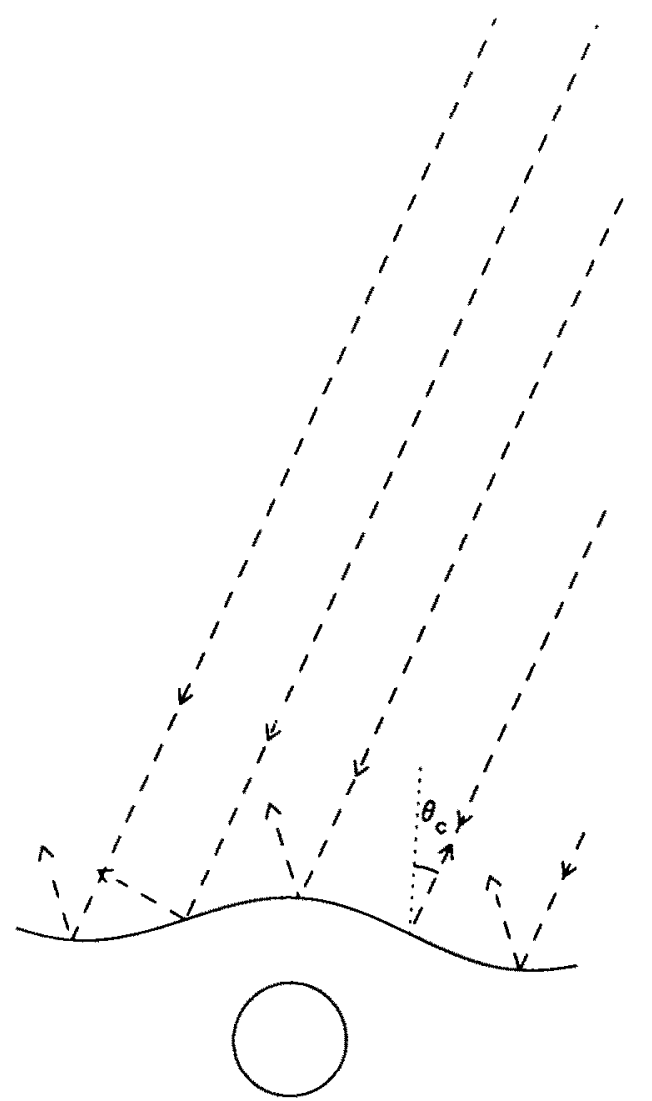

Fig. 4. Illustration of classical scattering from a rigid hard surface, where $\theta_{\mathrm{i}}=\theta_{\mathrm{c}}$, the direction of the surface normal tilted furthest from the vertical. Particles reflected from just one point in each unit cell will be directly backscattered.

appear in the final energy spectrum, as shown in fig. 5 . Note that such a direction will always be a rainbow angle, so the scattered intensity (integrated over a small detector width) will be relatively large.

Now we can discuss $\mathrm{He}$ scattering from LiF(001). Using the results of the previous two sections, we find, for the square of the energy width of a simple peak:

$$
\begin{aligned}
(\Delta E)^{2}= & {\left[g_{\perp}\left(\mu, \theta_{\mathrm{i}}, \theta_{\mathrm{f}}\right) \hbar \omega_{\perp}\right.} \\
& \left.+g_{\|}\left(\mu, \theta_{\mathrm{i}}, \theta_{\mathrm{f}}\right) \hbar \omega_{\|}\right] E_{\mathrm{i}} .
\end{aligned}
$$

The notation used is shown in fig. 6. $\theta_{\mathrm{i}}$ and $\theta_{\mathrm{f}}$ are the incident and final scattering angles, respec- 


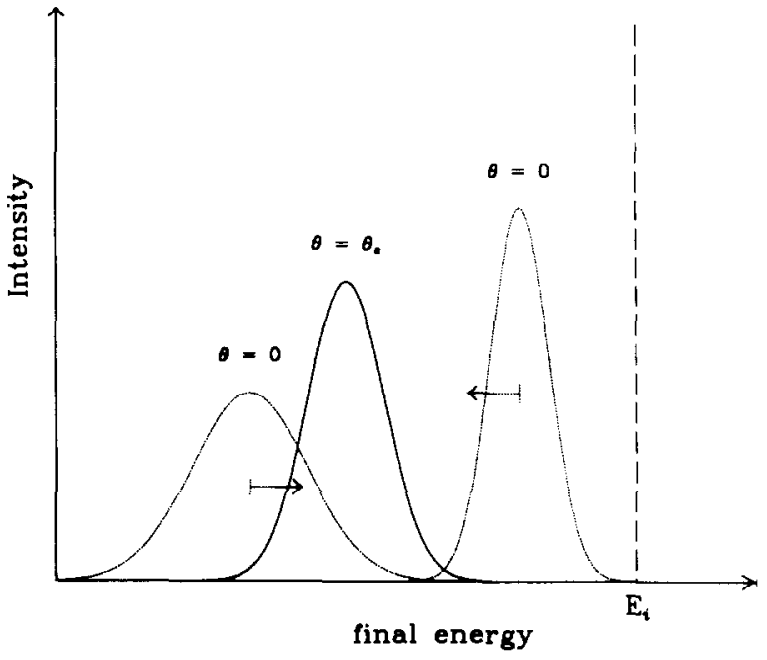

Fig. 5. Schematic final energy spectrum for direct backscattering at the largest incident angle at which scattered particles are detected. The dashed lines and arrows indicate how the spectrum changes as $\theta_{\mathrm{i}}$ increases from $0^{\circ}$ to this angle.

tively, and $\phi_{\mathrm{i}}$ and $\phi_{\mathrm{f}}$ are the azimuthal angles. The total scattering angle is denoted by $\theta_{t}\left(=\theta_{i}+\right.$ $\theta_{\mathrm{f}}$ ). In-plane scattering (to which we restrict ourselves) occurs when $\phi_{\mathrm{i}}=\phi_{\mathrm{f}}$ (we use negative values of $\theta_{\mathrm{f}}$ in place of $\phi_{\mathrm{f}}=\phi_{\mathrm{i}}+\pi$ ). In these coordi-

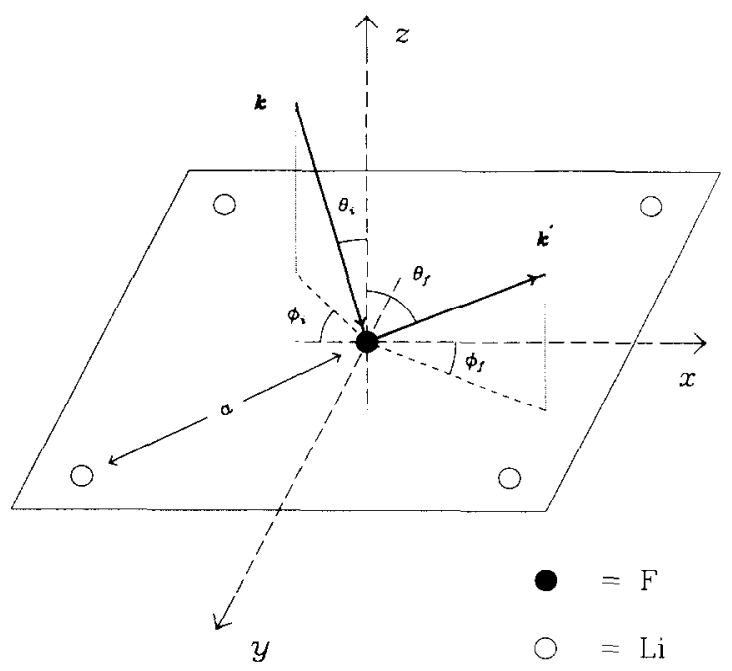

Fig. 6. Notation for angles, surface atoms and directions in the surface. nates the equations for the kinematic factors, defined by eqs. (6) to (8) become:

$$
\begin{aligned}
& g_{\perp}\left(\mu, \theta_{\mathrm{i}}, \theta_{\mathrm{f}}\right) \\
& =\frac{f\left(\mu, \theta_{\mathrm{t}}\right)}{\mu}\left\{\frac { \mu } { 1 + \mu } \left[\cos \theta_{\mathrm{f}}\right.\right. \\
& \left.\left.\quad+\frac{(1+\mu) \cos \theta_{\mathrm{i}}-\mu \cos \theta_{\mathrm{f}} \cos \theta_{\mathrm{t}}}{\sqrt{1-\mu^{2} \sin ^{2} \theta_{\mathrm{t}}}}\right]\right\}^{2}, \\
& g_{\|}\left(\mu, \theta_{\mathrm{i}}, \theta_{\mathrm{f}}\right) \\
& =\frac{f\left(\mu, \theta_{\mathrm{t}}\right)}{\mu}\left\{\frac { \mu } { 1 + \mu } \left[\sin \theta_{\mathrm{f}}-\left((1+\mu) \sin \theta_{\mathrm{i}}\right.\right.\right. \\
& \left.\quad+\mu \sin \theta_{\mathrm{f}} \cos \theta_{\mathrm{t}}\right) \\
& \left.\left.\quad \times\left(\sqrt{1-\mu^{2} \sin ^{2} \theta_{\mathrm{t}}}\right)^{-1}\right]\right\}^{2},
\end{aligned}
$$

where

$f\left(\mu, \theta_{\mathrm{t}}\right)=\left(\frac{\sqrt{1-} \mu^{2} \sin ^{2} \theta_{\mathrm{t}}-\mu \cos \theta_{\mathrm{t}}}{1+\mu}\right)^{2}$.

As $\left(\partial p_{\mathrm{f}} / \partial \boldsymbol{P}\right)_{\boldsymbol{P}=\mathbf{0}}$ vanishes by symmetry for directions perpendicular to the scattering plane [see eq. (8)], there is no contribution to the width due to fluctuations in this direction. We denote surface positions by $(x \sqrt{2} / a, y \sqrt{2} / a)$ where the origin is centered on an $\mathrm{F}$ atom, and the orientation of the axes is as shown in fig. 6; surface directions are defined with respect to these two axes. For example, there is a $\mathrm{Li}$ atom at $(1,1)$ and the line connecting it to $(0,0)$ points in the (11) direction.

Next we consider which peaks will be seen in the final energy spectrum. From the translational symmetry of the crystal surface, the final energy and direction of an atom scattered from a point $(x, y)$ in the surface will be identical to that of an atom scattered from any other point related to $(x, y)$ by a translation vector of the surface lattice. Thus we need only study points of incidence within one unit cell, which we choose to be the square with corners at $(1,1),(-1,1),(-1,-1)$ and $(1,-1)$. As a guide we again imagine classical scattering from a hard rigid surface identical to the elastic potential surface at the incident energy, as illustrated in fig. 3a. As the surface is fairly flat 
and we will not be considering large incident angles, the direction of the surface normal will determine the final scattered direction. Fig. 7 is a contour plot of $\theta_{n}$, the polar angle of the surface normal, in the unit cell. It vanishes at all maxima, minima and saddle points of the surface, and has four maxima itself, as indicated in the figure. From symmetry, the value of $\theta_{\mathrm{n}}$ is the same at each of these maxima. We denote this maxima $\theta_{\mathrm{n}}$ by $\theta_{\mathrm{c}}$ (the critical angle), and the four values of $\phi_{\mathrm{n}}$ are $0, \frac{1}{2} \pi, \pi, \frac{3}{2} \pi$. For direct backscattering from this surface at $\theta_{i}=\theta_{c}$ and in one of these azimuthal planes, only one peak will be seen. For inelastic scattering from the real surface, the same considerations imply that this statement is still true, although the value of $\theta_{c}$ will, in general, be different.

Fig. 8 is an estimated final energy spectrum for He striking $\mathrm{LiF}(001)$. The peak in the loss spectrum is due to a single collision with a $F$ atom. The scattering is in-plane along the (10) direction. The incident beam has an energy of $0.5 \mathrm{eV}$. The detector is set for direct backscattering, $\theta_{\mathrm{f}}=-\theta_{\mathrm{i}}$.

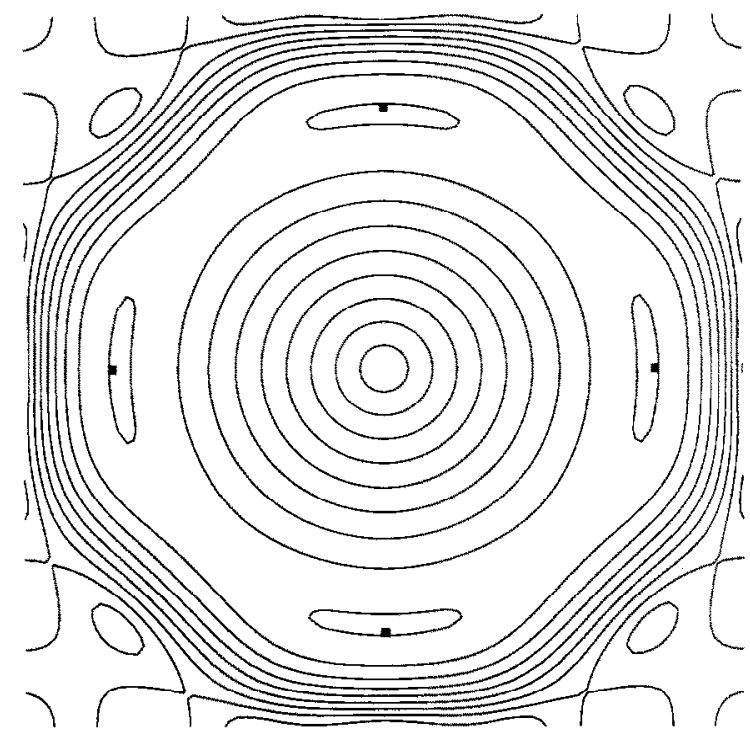

Fig. 7. Contour plot of the polar angle of the normal to the equipotential surface shown in fig. 3. A F atom is in the center, while there is a $\mathrm{Li}$ atom in each corner. Directly above each atom $\theta_{\mathrm{n}}$ vanishes. The black squares indicate the positions of $\operatorname{maximum} \theta_{\mathrm{n}}$.

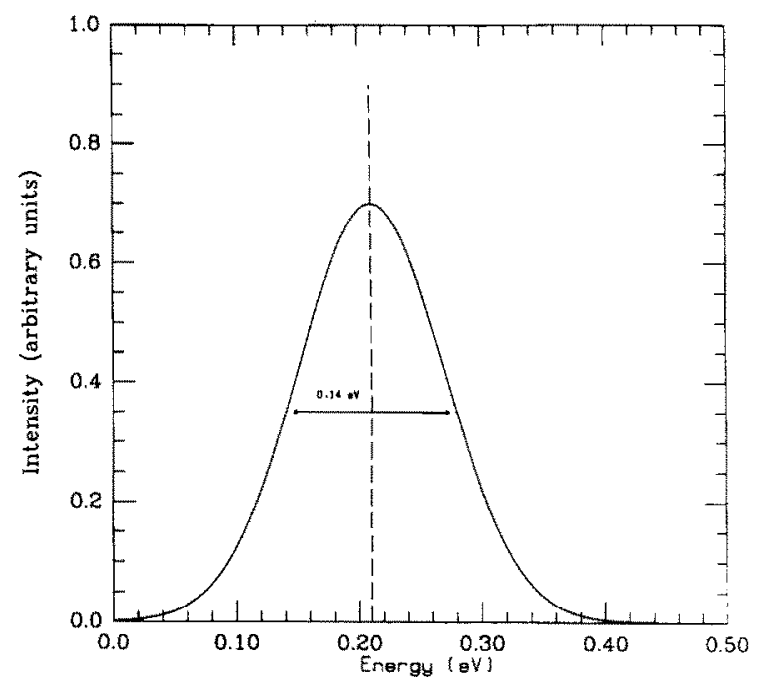

Fig. 8. Calculated final energy spectrum for $0.5 \mathrm{eV}$ He directly backscattered from LiF in the (10) plane and at the largest incident angle for which scattered particles may be detected.

The surface is at a low temperature (see section 5). We have calculated the width assuming $\theta_{\mathrm{c}} \approx 27^{\circ}$, the critical angle for elastic scattering from the equipotential surface shown in fig. $3 a$. For the real inelastic scattering, this angle will be different, but the final energy spectrum (at the real $\theta_{c}$ ) will be approximately the same; for direct backscattering, the classical final energy is independent of the incident angle (given our assumptions), while the width is also insensitive to $\theta_{\mathrm{i}}$ for an oscillator as nearly isotropic as $\mathrm{F}$ in $\mathrm{LiF}$ (see table 2). Again we expect relatively large intensity, as this angle is a classical rainbow angle. We estimate the error in energy loss due to interaction with the F atom's neighbors to be less than $15 \%$.

In practice, the source and detector cannot be completely aligned so that, for in-plane scattering, we must have a finite total scattering angle. This, however, does not invalidate our conclusion that, for a fixed total scattering angle, there will exist a critical incident angle at which just one peak is seen, and beyond which, the intensity vanishes (provided we continue to avoid multiple scattering and shadowing). Fig. 9 shows an estimated final energy spectrum for the $F$ peak of fig. 8 , with a different source-detector angle. This has been calculated from eqs. (14) to (17) assuming (a) that 


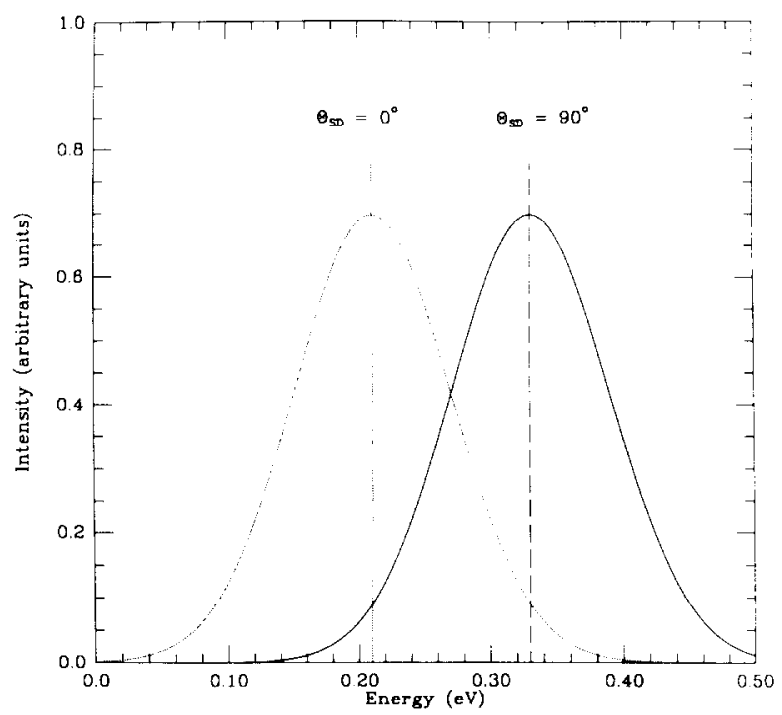

Fig. 9. Calculated final energy spectra for different total scattering angles. The conditions are the same as for fig. 8 , except that the total scattering angle is varied.

the point of inflection (strictly, the point of zero Gaussian curvature) of the equipotential surface is the impact point and (b) that the normal here passes through the equilibrium position of the $F$ atom [25]. From these calculations, for a sourcedetector angle of $90^{\circ}$, the final scattered angle will be $\theta_{\mathrm{f}}=79^{\circ}$, so multiple collisions may occur in this case.

As long as only one peak appears or, if more than one, that a simple peak is resolvable, we may use eqs. (14) to (17) to predict its dependence on the adjustable experimental parameters. The mean final energy is linear in the incident energy, while the width varies as its square root. In varying the incident energy, however, one must be careful not to reduce it below the range of validity of the impulsive approximation or the semiclassical theory. Furthermore, the complete angular dependence is given by the kinematic factors $g_{\perp}$ and $g_{\|}$, although their variation may be small over the range of angle in which this peak is resolvable. We may also deduce the qualitative nature of the final energy spectrum as a function of $\theta_{\mathrm{i}}\left(=-\theta_{\mathrm{f}}\right)$ and $\phi_{\mathrm{i}}\left(=\phi_{\mathrm{f}}\right)$ from our rigid hard wall model. To begin, we align the scattering plane along (10) and keep $\theta_{\mathrm{i}}$ large. We rotate the plane of the surface about (01) (i.e., reduce $\theta_{i}$ ) until a peak appears in the final energy spectrum. Just at this point, rotations of the scattering plane (i.e., changes in $\phi_{i}$ ) will make the peak disappear. As $\theta_{i}$ is further decreased, this one peak will widen, eventually splitting in two, while, for a fixed $\theta_{i}$, rotations of the scattering plane will decrease its width. However, as $\theta_{i}$ is reduced, other peaks, due to scattering from different points in the unit cell, will appear, and may obscure these features. Eventually, near normal incidence and reflection, there will be several peaks, one of which will correspond to simple collisions with the $\mathrm{Li}$ atom which, if it can be isolated, will obey eqs. (14) to (17) also. Although it is beyond the scope of the present work, in principle, the entire spectrum and its angular dependence are calculable [14].

We conclude this section with a discussion of another possible surface on which this trial experiments might be performed, and which illustrates other features. Fig. $3 \mathrm{~b}$ is an equipotential surface for $0.5 \mathrm{eV}$ He scattering from $\mathrm{CsF}(001)$. The larger hills correspond to the $\mathrm{Cs}$ ions, so that the single peak visible at the critical polar angle is due to simple collisions with these ions. The left peak in fig. 10 is an estimated final energy spectrum for a

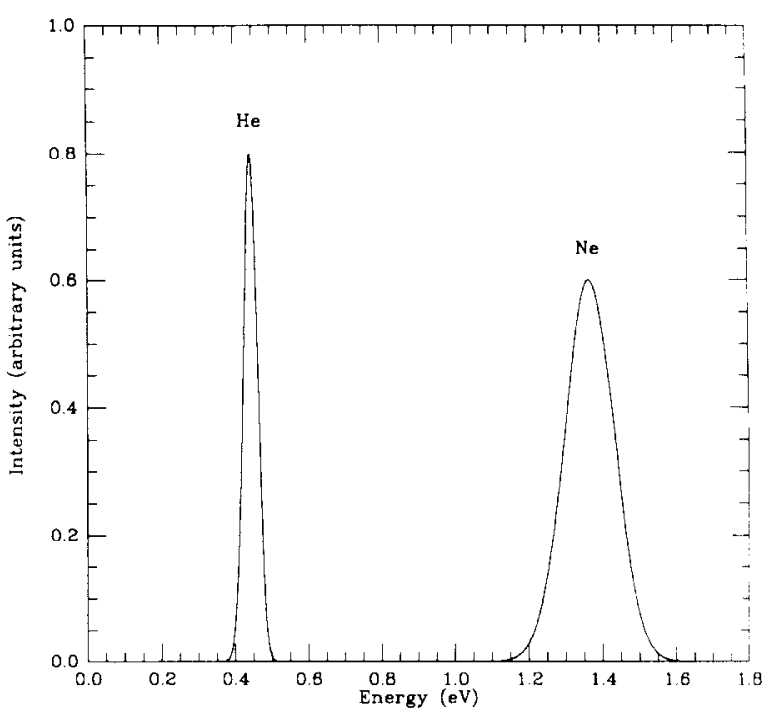

Fig. 10. Estimated final energy peaks for $\mathrm{He}$ and $\mathrm{Ne}$ backscattered from CsF. The scattering is in the (01) plane and at the largest incident angle for which scattered particles may be detected. The initial energy of the $\mathrm{He}$ is $0.5 \mathrm{eV}$, and of the $\mathrm{Ne}$ is $2.5 \mathrm{eV}$. 
$0.5 \mathrm{eV} \mathrm{He}$ beam directly backscattered from $\mathrm{CsF}(001)$ at the critical polar angle, along the (01) direction. The scattering is very close to elastic, due to the small ratio of the $\mathrm{He}$ and Cs masses. If the beam now seeded with $\mathrm{Ne}$, and the crystal is rotated (about (10)) to the new critical angle, the spectrum shown by the right peak in fig. 10 results. As seeding produces a $\mathrm{Ne}$ beam with the same initial velocity as the $\mathrm{He}$, the incident energy is $2.5 \mathrm{eV}$. A similar spectrum could be found for Ar seeding. Thus the dependence of peak width on the mass ratio, for fixed initial velocity, may be tested. Finally, in fig. 11, we show again $0.5 \mathrm{eV} \mathrm{He}$ on $\mathrm{CsF}(001)$, but now at normal incidence and detection. We expect four peaks in this case: the two simple peaks due to collisions with $\mathrm{Cs}$ and $\mathrm{F}$, an almost elastic peak due to reflection from the global minima, and a peak due to reflection from the saddle points. These last two are shown only schematically. Thus it may be possible, for this system, to identify and measure the width of the peak due to simple collisions with the smaller ion also.

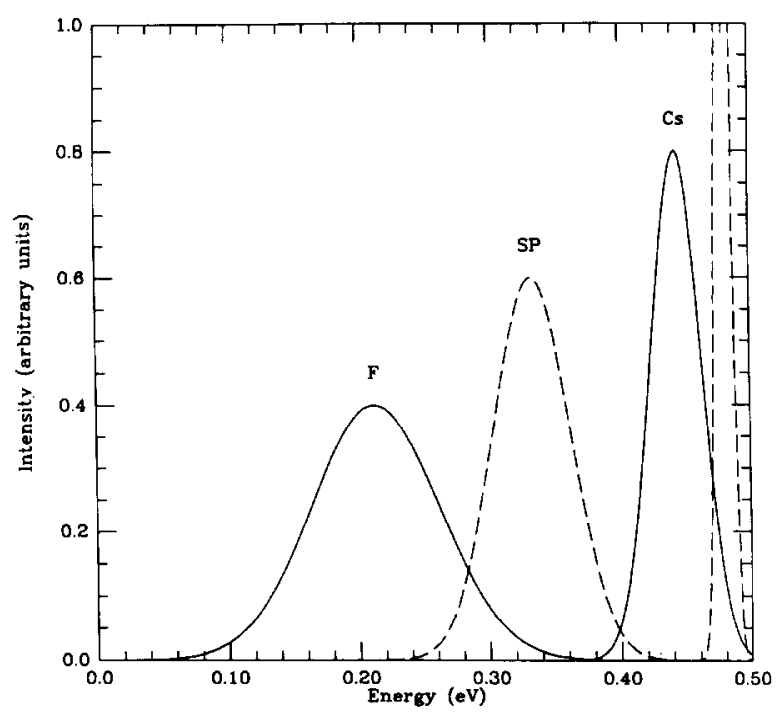

Fig. 11. Estimated final energy spectrum for $0.5 \mathrm{eV} \mathrm{He}$ on $\mathrm{CsF}(001)$ at normal incidence and reflection. The two solid peaks are due to simple collisions with the $\mathrm{Cs}$ and $\mathrm{F}$, while the dotted ones are schematics based on crude estimates.

\section{Extension to finite surface temperatures}

Finally we consider the temperature dependence of the widths of these peaks. Eq. (1) is also expected to be accurate for surfaces at finite temperatures, provided $k_{\mathrm{B}} T_{\mathrm{s}} \ll E_{\text {loss }}$, where $T_{\mathrm{s}}$ is the surface temperature [14]. This condition is easily satisfied by surfaces at room temperature $\left(k_{\mathrm{B}} T_{\mathrm{s}} \approx\right.$ $25 \mathrm{meV}$ ) for the systems discussed here. For a simple peak the only change with temperature is an increase in the size of the momentum fluctuations of the surface atom. For a single oscillator we have

$$
\left\langle\hat{P}^{2}\right\rangle=\left(\frac{1}{2} \hbar m \omega\right) \operatorname{coth}\left(\frac{\hbar \omega}{2 k_{\mathrm{B}} T}\right) .
$$

Here $\hat{P}$ is the momentum operator, $m$ the mass, $\omega$ the frequency and $T$ the temperature of the oscillator. From eq. (14), we find that the width, as a function of $T_{\mathrm{s}}$, is given by

$$
\begin{aligned}
(\Delta E)^{2}= & {\left[g_{\perp}\left(\mu, \theta_{\mathrm{i}}, \theta_{\mathrm{f}}\right) \hbar \omega_{\perp} \operatorname{coth}\left(\frac{\hbar \omega_{\perp}}{2 k_{\mathrm{B}} T_{\mathrm{s}}}\right)\right.} \\
& \left.+g_{\|}\left(\mu, \theta_{\mathrm{i}}, \theta_{\mathrm{f}}\right) \hbar \omega_{\|} \operatorname{coth}\left(\frac{\hbar \omega_{\|}}{2 k_{\mathrm{B}} T_{\mathrm{s}}}\right)\right] E_{\mathrm{i}} .
\end{aligned}
$$

This function is plotted against temperature in fig. 12. The parameters are those for the peak shown in fig. 8 , but now with the surface at finite temperature. At low temperatures $\left(T_{\mathrm{s}} \ll \hbar \omega_{\mathrm{D}}\right)$ the width is dominated by quantum effects. In fact, for temperature up to $50 \mathrm{~K}$ the fluctuations are still almost entirely quantum mechanical. When we go to high temperatures thermal broadening will dominate the quantum effects, and the peak itself may become difficult to identify. At room temperature the width is practically proportional to $T_{\mathrm{s}}$, and this dependence has been seen experimentally by Hurst et al. [26] in the scattering of Ar from tungsten. However, they did not perform measurements at temperatures sufficiently low to see the quantum fluctuations. From eq. (1) it is clear that the finite (quantum mechanical) value at $T_{\mathrm{s}}=0$ and the linear behavior at high $T_{s}$ are qualitative features of all peaks, not just of the type we have been studying. 


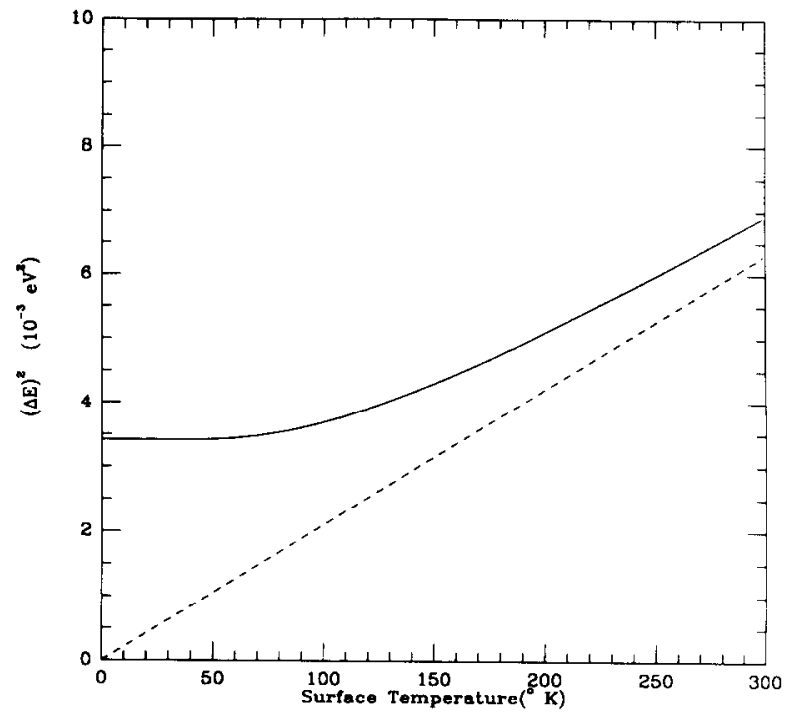

Fig. 12. The width of the peak, shown in fig. 8 at zero temperature, as a function of surface temperature (solid line). The dashed line indicates the classical behaviour.

Available techniques should be sufficient for determining this zero point motion effect. Both the source and the detector need to operate at energies of $0.5 \mathrm{eV}$ to ensure that peaks can be made sufficiently narrow for the semiclassical theory to apply. The resolution of final energies and angles attainable with a velocity selector at thermal energies will suffice. (As these peaks contain a large fraction of the outgoing intensity, the loss of intensity involved in using a velocity selector should not be a problem.) The effects of thermal broadening will be relatively small below $\sim 50 \mathrm{~K}$. Other desirable features of such an experiment include the ability to rotate the crystal and to vary the surface temperature. Finally, the source-detector angle should not be fixed at too large a value.

\section{Discussion}

The success of this prototype experiment would provide justification for more detailed calculations and further experimental investigation. The technique will yield information complimentary to that found in thermal energy experiments; it will probe directly the dynamics of individual atoms, rather than the motion of normal modes. The full classical map of impact parameters and initial momenta to final momenta can be calculated by numerical simulation, once the potentials involved are known. This can be used to identify situations where isolated peaks occur, whose widths may be found from eq. (1). It may be possible to probe all points on the surface unit cell in this manner, from a variety of different incident angles. For atom-surface collisions which are not so impulsive (e.g., noble gases on metals), there will be larger contributions from the interatomic correlation functions appearing in eq. (1). Thus, several force constants may be determined. By measuring these local force constants, we can make direct comparisons with models of interatomic surface forces. These may differ considerably from those in the bulk, even, as we showed, in the simple Born-Mayer scheme.

The new technique will provide, at the very least, constraints on theories of interatomic forces. A case in point concerns the frequency of the Rayleigh mode in $\operatorname{LiF}(001)$ at the $\overline{\mathrm{M}}$-point of the surface Brillouin zone. Models of the interatomic forces, fitted to bulk data, yield a value about $10 \%$ higher than that determined by scattering experiments performed at thermal energies. This discrepancy can be removed by assuming various modifications in the properties of the ions at the surface [27]: increased $\mathrm{F}^{-}$polarizability, different non-central nearest-neighbor transverse forces, or the charge overlap effect. Each modification may give rise to different widths of the final energy peaks in the experiment suggested above, and accurate measurements of these widths (combined with numerical calculations) may provide a criterion for choice between the various models.

To conclude, hyperthermal atom-surface scattering behaves almost classically. At some rainbow angles, the final energy spectrum can be reduced to one (or more) easily identified peaks. The zero point vibrations of surface atoms cause a broadening of these peaks. This is a quantum mechanical effect, and provides a new sight into surface dynamics. In this paper, we studied impulsive two-body collisions, and derived an analytic result for the width of an energy peak in this casc. As an example, we made semi-quantitative predictions 
for He scattering from LiF. Our general formalism applies to many physical systems and may provide a useful method for obtaining information about a surface's dynamical properties. Experimental test of our predictions would provide justification for more detailed calculations.

\section{Acknowledgements}

We are very grateful to Horia Metiu for helpful conversations. This work has been supported by NSF grant DMR87-03434 and ONR grant N00014-84-K-0548.

\section{Appendix}

To estimate the vibration frequencies of the atoms in an alkali halide crystal with the $\mathrm{NaCl}$ structure we use the Born-Mayer scheme. We look at both bulk and surface frequencies and compare our results with those of detailed slab calculations.

The ions are taken to interact through both long-range Coulomb forces and a short range exponential repulsion between nearest neighbors only,

$$
V(r)=A \exp (-r / \rho)
$$

The values of $A$ and $\rho$ may easily be related to the bulk modules $B$ and the equilibrium interionic spacing $a$ for the material [15]. In particular, the decay constant, $\rho$, obeys the relation

$a / \rho=2+18 B a^{4} /\left(\alpha e^{2}\right)$,

where $\alpha$ is the Madelung constant and $e$ is the electronic charge. To find the bulk frequencies, we consider the restoring forces on an ion displaced slightly from its equilibrium position, holding the other atoms fixed. Cubic symmetry and Laplace's equation require the Coulomb contribution to vanish, and from the Born-Mayer repulsion we find

$\omega_{\mathrm{B}}=\sqrt{6 \mathrm{Ba} / \mathrm{m}}$,

where $\omega_{B}$ is the bulk frequency of the atom, and $m$ is its mass. Comparison of this frequency for the lighter atom with the measured optical frequency at $k=(\pi / a, 0,0)$ yields an error of no more than $30 \%$ for any alkali halide with $\mathrm{NaCl}$ structure.

The surface frequencies are found in a similar fashion. The small relaxation that occurs has a negligible effect on the frequencies [28], and can be ignored. Now the Coulomb contribution does not vanish. A simple calculation gives:

$\omega_{\perp}=\left(\frac{\omega_{\mathrm{B}}}{\sqrt{2}}\right) \sqrt{(a / \rho-4+3 I / \alpha) /(a / \rho-2)}$,

and

$\omega_{\|}=\omega_{\mathrm{B}} \sqrt{(a / \rho-3 / 2-3 I / 4 \alpha) /(a / \rho-2)}$,

where

$I \equiv \sum_{m, n}^{\prime} \frac{(-1)^{(m+n+1)}}{\left(m^{2}+n^{2}\right)^{3 / 2}}$.

The sums over $m$ and $n$ above range from $-\infty$ to $+\infty$, excluding the pair $m=0, n=0$. The sum may be easily performed [29], and $I \simeq 2.646$. Note that for very steep repulsive forces $(\rho<a), \omega_{\perp} \sim$ $\omega_{\mathrm{B}} / \sqrt{2}$ and $\omega_{\|} \sim \omega_{\mathrm{B}}$.

Table 2 shows results for the crystals mentioned in this paper. The restoring forces are close to isotropic in all cases, and the frequencies are in the range $10^{13}-10^{14} \mathrm{rad} / \mathrm{s}$. We also show frequencies calculated from the mean square velocities at zero temperature given by Chen et al. [23] for LiF. Their values were obtained from studies of the complete lattice dynamics. We use these in our width estimates. For the six alkali halides for which Chen et al. give figures, the above formulae are accurate to within $20 \%$.

\section{References}

[1] A. Lock, J.P. Toennies, Ch. Wöll, V. Bortolani, A. Franchini and G. Santoro, Phys. Rev. B 37 (1988) 7087.

[2] G. Benedek, J.P. Toennies and R.B. Doak, Phys. Rev. B 28 (1983) 7277.

[3] N. Garcia, J. Chem. Phys. 67 (1977) 897.

[4] D. Eichenhauer and J.P. Toennies, J. Chem. Phys. 85 (1986) 532. 
[5] J.P. Toennics, in: Solvay Conference on Surface Science, Ed. F.W. de Wette (Springer, New York, 1988) p. 248.

[6] For a review, see: H. Hoinkes, Rev. Mod. Phys. 52 (1980) 933.

[7] A. Amirav, M.J. Cardillo, P.L. Trevor, C. Lim and J.C. Tully, J. Chem. Phys. 87 (1987) 1796.

[8] C. Lim. J.C. Tully, A. Amirav, P.L. Trevor and M.J. Cardillo, J. Chem. Phys. 87 (1987) 1808.

[9] R. Brako and D.M. Newns, Surf. Sci. 117 (1982) 43.

[10] J.D. McClure, J. Chem. Phys. 52 (1970) 2712.

[11] See, for example, E. Hulpke, Surf. Sci. 52 (1975) 615; U. Gcrlach-Mcycr, E. Hulpke and D.H. Meyer, Chem. Phys. 36 (1979) 327.

[12] R.L. McEachern et al., Rev. Sci. Instrum. 59 (1988) 2560; A.D. Tenner, K.T. Gillen, T.C.M. Horn, J. Los and A.W. Kleyn, Surf. Sci. 172 (1986) 90;

E. Hulpke and K. Mann, Surf. Sci. 133 (1983) 171.

[13] J. Böheim and W. Brenig, Z. Phys. B (Condensed Matter) 41 (1981) 243.

[14] J.H. Jensen, P. Chang and W. Kohn, Phys. Rev. A 40 (1989) 1198;

J.H. Jensen and W. Kohn, Phys. Rev. A 40 (1989) 2309.

[15] See, for example, J.M. Ziman, Theory of Solids (Cambridge University Press, Cambridge, 1972) p. 129.

[16] There is also a shift in the mean energy of the peak away from its classical value, but this will be more difficult to detect, as it is of higher order in $\hbar$ (namely $\mathrm{O}(\hbar)$ ).

[17] See, for example, the work of McClure [10] for a classical solution and that of Chen et al. [23] for surface dynamics.

[18] N.W. Ashcroft and N.D. Mermin, Solid State Physics (Holt, Rinehart and Wilson, Philadelphia, 1976) p. 459.

[19] For an alkali halide crystal, the surface atoms are in fact either alkali or halide ions.
[20] P.W. Fowler and J.M. Hutson, Phys. Rev. B 23 (1986) 3724.

[21] See, for example, H. Inouye and S. Kita, J. Chem. Phys. 56 (1972) 4877;

S. Kita, K. Noda and H. Inouye, J. Chem. Phys. 63 (1975) 4930.

[22] See, for example, R.G. Gordon and Y.S. Kim, J. Chem. Phys. 56 (1972) 3122;

Y.S. Kim and R.G. Gordon, J. Chem. Phys. 60 (1974) 4323.

[23] T.S. Chen, G.P. Alldredge and F.W. de Wette, Surf. Sci 57 (1976) 25 .

[24] See, for example, E. Hulpke, Surf. Sci. 52 (1975) 615;

A. Harkmans, E.G. Overbosch and J. Los, Surf. Sci. 62 (1977) 621;

E. Hulpke and K. Mann, Surf. Sci. 157 (1985) 157.

[25] In fact, the angle between the line from the point of impact to the equilibrium position of the $F$ atom and the normal is $7^{\circ}$.

[26] J.E. Hurst, C. Wharton, K.C. Janda and D.J. Auerbach, J. Chem. Phys. 78 (1983) 1559.

[27] W. Kress, F.W. de Wette, A.D. Kularni and U. Schröder. Phys. Rev. B 35 (1987) 2467.

[28] W. Kress, F.W. de Wette, A.d. Kularni and U. Schröder, Phys. Rev. B 35 (1987) 5783.

[29] F.W. de Wette, W. Kress and U. Schröder, Phys. Rev. B 32 (1985) 4143.

[30] S. Kita, K. Noda and H. Inouye, J. Chem. Phys. 64 (1976) 3446.

[31] H. Inouye, K. Noda and S. Kita, J. Chem. Phys. 71 (1979) 2135.

[32] H. Inouye and S. Kita, J. Chem. Phys. 72 (1980) 1301.

[33] Y.S. Kim and R.G. Gordon, J. Chem. Phys. 61 (1974) 1. 